\title{
Vibration Response of Cracked Cantilevered Plates Near Natural Frequency Veerings
}

\author{
Akira Saito* and Matthew P. Castanier ${ }^{\dagger}$ \\ Department of Mechanical Engineering \\ The University of Michigan, Ann Arbor, MI 48109-2125, USA \\ Christophe Pierre $\ddagger$ \\ Faculty of Engineering \\ McGill University, Montreal, Quebec, H3A 2K6, Canada
}

\begin{abstract}
In this paper, the linear and nonlinear vibration response of a cracked cantilevered plate is investigated as the crack location or crack length is varied. Of particular interest is the vibration response in parameter regions where the natural frequency loci show veerings. For a representative finite element model, it is shown that the veerings due to crack length variation involve the switching of mode shapes and modal interactions. The nonlinearity caused by the crack closing effect is then introduced, and its effect on the vibration response near the veerings is discussed. A hybrid-interface method of component mode synthesis is employed to generate a compact reducedorder model of the system while retaining the crack surface degrees of freedom (DOF) as physical DOF so that the nonlinear boundary conditions caused by the contact may be enforced. The nonlinear forced response analysis is carried out using a hybrid frequency/time domain method, which is based on the method of harmonic balance. The nonlinear vibration response near loci veerings and crossings due to the variation of crack length is investigated in detail. Furthermore, an alternative method for estimating the nonlinear resonant frequency is introduced by extending the bilinear frequency approximation, and its advantages and limitations are addressed.
\end{abstract}

\section{Introduction}

$\mathrm{I}$ is well known that the natural frequencies of cracked elastic structures differ from their healthy counterparts. A comprehensive literature survey of research activities regarding the vibration problems of various structures with cracks is found in the work by Dimarogonas. ${ }^{1}$ In this paper, linear and nonlinear vibration of a cantilevered rectangular plate with a crack is investigated. The primary focus of this study is the vibration response near the eigenvalue loci veerings and crossings that occur as the crack length or location is varied.

Eigenvalue loci veerings, also known as avoided crossings, or eigenvalue avoidance, are observed in plots of eigenvalues versus a system parameter. In particular, a veering refers to a region in which two eigenvalue loci approach each other and almost cross as the system parameter is changed, but instead of crossing they appear to veer away from each other, with each locus then following the previous path of the other. ${ }^{2}$ Although this phenomenon was initially regarded as an "aberration" caused by approximation methods applied to the original infinite-dimensional eigenvalue problems, ${ }^{3}$ it was shown by Perkins and Mote ${ }^{4}$ that the phenomenon can be observed for continuous systems. Since then, several researchers have noted and investigated the relation between veerings and mode localization phenomena. ${ }^{5-7}$ In conjunction with

\footnotetext{
${ }^{*}$ Graduate Student Research Assistant, Student Member of AIAA

${ }^{\dagger}$ Associate Research Scientist, Senior Member of AIAA

${ }^{\ddagger}$ Dean, Senior Member of AIAA
} 
the localization, it is known that the veerings are associated with coupling between the modes, which is typically seen as the coupled mode shapes near the veering regions or even between modes in different physical domains, such as electrical and mechanical domains. ${ }^{8}$ These phenomena have also been investigated for the damaged structures, such as two-span weakened column, ${ }^{9}$ and cables with damage. ${ }^{10}$

For vibration problems of cracked rectangular plates, variations in natural frequencies and mode shapes due to crack length variations have been known for a long time. The initial contribution to the study of vibration problems of cracked rectangular plates was made by Lynn and Kumbasar, ${ }^{11}$ who calculated the vibration frequency drop of plates due to cracking by numerically solving the Fredholm integral equation of the first kind. Petyt ${ }^{12}$ also investigated the variation of frequency of fundamental mode due to crack length by experiments and a finite element method. Those contributions were followed by a number of investigations based on plate vibration theory, including those by Stahl and Keer, ${ }^{13}$ Hirano and Okazaki, ${ }^{14}$ Solecki, ${ }^{15}$ and Yuan and Dickinson. ${ }^{16}$ Although the trajectories of frequencies versus crack length appear in these articles, the veering regions and associated dynamics of the cracked plates near those regions were not highlighted. Liew et al. ${ }^{17}$ applied a domain decomposition method to obtain the out-of-plane vibration frequencies of cracked plates, and they not only confirmed the results found by Stahl and Keer ${ }^{13}$ and Hirano and Okazaki ${ }^{14}$ but also considered a wider range of crack length ratio. It is noted that they examined a plate with a centrally-located internal crack and reported frequency crossings instead of veerings. In other words, for this case they observed that two approaching eigenvalue loci would intersect as crack length increased, which is also known as crossover. More recently, Ma and Huang ${ }^{18}$ also reported variations in natural frequencies and associated mode shapes due to changes in crack length for a square plate with an edge crack, based on experiments and finite element analysis. As was mentioned by many others, Ma and Huang stated that the nonlinearity due to the crack closing effect has to be considered for the in-plane bending case, but crack closing was neglected in their study because their work focused on the out-of-plane bending vibration.

In the studies of cracked rectangular plate vibrations reviewed above, the in-plane bending vibration was not considered and thus the crack closing effect was not examined. In contrast, the issue of crack closing effect naturally arose in the studies of vibration problems of cracked beams, for which in-plane bending vibration is typically of primary research interest. For the study of cracked Bernoulli-Euler beams, a pioneering contribution was made by Christides and Barr in their application of the Hu-Washizu-Barr variational principle to the cracked beam problem. ${ }^{19}$ Further extension was made by Shen and Pierre for BernoulliEuler beams with symmetric cracks ${ }^{20}$ and single-edge cracks. ${ }^{21}$ A generalization to the theory was made by Chondros et al. ${ }^{22}$ However, in these studies, the nonlinear effect was not considered. Gudmundson ${ }^{23}$ pointed out that measured natural frequencies of a beam with a fatigue crack differ from those calculated without considering the crack closing effect. He also addressed the significance of the crack closing effect for accurately predicting the frequency shifts due to cracking. The crack closing effect is also known to cause phenomena that appear only in nonlinear response cases, such as superharmonic and subharmonic resonances $^{24,25}$ and period doubling bifurcations. ${ }^{26,27}$

One of the methods to estimate the (primary) resonant frequencies of the cracked beams is the application of the bilinear frequency approximation. This was initially introduced for calculating the effective resonant frequencies of piecewise linear oscillators (e.g., Shaw and Holmes ${ }^{28}$ ), and it has been used for approximating the effective vibration frequency of multi-DOF piecewise linear systems (e.g., Butcher ${ }^{29}$ ). It has also been used for estimating the natural frequency of cracked beams. ${ }^{27,30,31}$ Chati et al. ${ }^{32}$ extended the concept of the bilinear frequency to study the vibration of a cracked beam using a multi-DOF oscillator model. They assumed that if the crack is sufficiently shallow, the actual and bilinear mode shapes are close to each other, and thus the frequency can be approximated by the bilinear frequency. Most of the methods reviewed above assume that the crack has only two states—closed or open. This assumption is accurate when the relative motion of the crack surfaces is simple, such as the in-plane bending vibration of cantilevered beams. However, in general, the motion of crack surfaces is more complicated, and there may be more than two states. For example, crack closing may proceed gradually and/or occur at different regions on the crack 
surfaces at different times.

The closing crack was also modeled by equivalent linear model by Kisa and Brandon, ${ }^{33}$ with the assumption that the stiffness change due to a crack can be expressed as a linear combination of the stiffness matrix of uncracked beam and that due to cracking and contact. An emerging approach for dealing with this issue is the application of Nonlinear Normal Modes. ${ }^{34-36}$ However, the applicability of this approach is still limited to simple structures or simplified vibration problems, due mostly to its computational costs for constructing the nonlinear normal modes.

With regard to the veering phenomena for nonlinear structural systems, very little is known about how the nonlinearities influence the response near the veering regions. Lacarbonara et al. ${ }^{37}$ investigated nonlinear modal interactions of an imperfect beam near veering regions, the nonlinearities of which are quadratic and cubic nonlinearities due to large-amplitude vibration, through perturbation and bifurcation analyses. They observed distinguishing features in the response, such as mode localization due to nonlinear coupling and frequency-island generation, which illustrates the richness of the dynamics in veering regions for nonlinear structural systems.

In this paper, the vibration of cracked cantilevered plates in frequency veering regions is investigated. As reviewed above, veering phenomena have not been studied thoroughly for cracked structures, in either the linear or nonlinear dynamics regime. Regarding the vibration of cantilevered cracked plates, the research reviewed above focused only on the out-of-plane vibration, and crack closing effects were intentionally neglected. On the other hand, studies of cracked beams have focused on in-plane bending in most cases. Thus, the crack closing effect on the vibration response has been investigated in many studies of cracked beams. However, veering and modal interaction phenomena between in-plane and out-of-plane vibration modes have not been studied in this context. Moreover, in general, the veering phenomena in nonlinear structural systems have not been studied well. Therefore, in this paper, first the eigenvalue loci veering due to cracking is shown using a cracked cantilevered plate example without considering the crack closing effect. The crack closing effect is then included and associated nonlinear resonant frequencies are identified. Furthermore, the bilinear frequency approximation is extended such that the linear free response analysis results can be used to estimate the resonant frequencies of the nonlinear forced response near veerings. The effects of the crack closing on the resonant frequencies are discussed in detail for some specific veering regions.

This paper is organized as follows. In section II, the cracked plate vibration problem and the finite element model are introduced. In section III, the linear free response of a cracked plate is considered using a finite element model of a three-dimensional cantilevered plate with a planar surface-breaking crack that runs parallel to the cantilevered edge, and the associated frequency veering and crossing phenomena are shown. In section IV, a solution technique for the nonlinear forced response analysis, called the hybrid frequency/time (HFT) method, is briefly reviewed. The nonlinear forced response calculation is then carried out and the effects of nonlinearity to the response in the neighborhood of representative veering regions are discussed in detail. In section $\mathrm{V}$, an alternative method for estimating the nonlinear resonant frequency is introduced as an extension to the bilinear frequency approximation. The results are compared with the results obtained with the forced response analysis, and the advantages and the limitations of the bilinear frequency approximation are discussed. Finally, conclusions are summarized in section VI. 


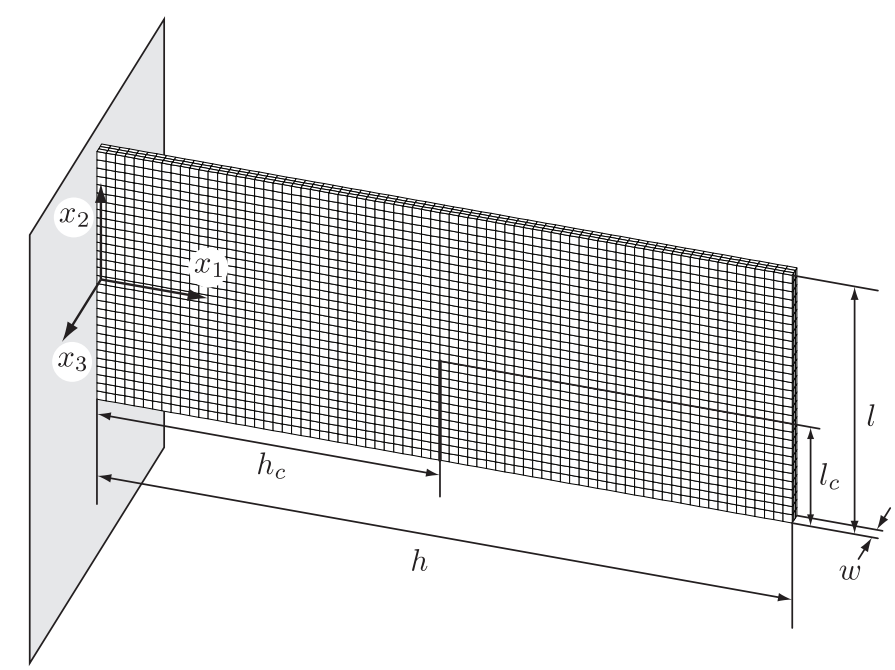

Figure 1. Finite element model of the cracked plate

\section{Cracked Plate Model}

In this paper, the vibration of a cantilevered rectangular plate comprised of linear isotropic elastic material is considered. Let $\Omega \in \mathbb{R}^{3}$ denote the domain of the plate fixed on a portion of its boundary, and assume the material undergoes infinitesimal deformation, and finite rotations are not permitted. The elastodynamic problem is then formulated through the variational formulation, followed by the discretization using Galerkin's method. Namely, the semi-discrete governing equation of the cracked plate is formulated as

$$
\mathbf{M} \ddot{\mathbf{u}}(t)+\mathbf{C} \dot{\mathbf{u}}(t)+\mathbf{K u}(t)=\mathbf{b}(t)+\mathbf{f}(\mathbf{u}) ; \quad \mathbf{M}, \mathbf{C}, \mathbf{K} \in \mathbb{R}^{n \times n}, \mathbf{u}, \mathbf{b}, \mathbf{f} \in \mathbb{R}^{n}
$$

where $\mathbf{u}$ is the displacement vector, $\mathbf{M}, \mathbf{C}$ and $\mathbf{K}$ denote the mass, damping, and stiffness matrices, $\mathbf{b}(t)$ denotes the time-dependent external force, and $\mathbf{f}(\mathbf{u})$ denotes the displacement-dependent nonlinear force, which in this case is the nonlinear force caused by the intermittent contact at the crack.

A finite element (FE) model of a cantilevered plate with a transverse crack is shown in Fig. 1, where $h=1.5 \times 10^{-1} \mathrm{~m}, l=6.0 \times 10^{-2} \mathrm{~m}, t=3.0 \times 10^{-3} \mathrm{~m}$. The material model is steel with Young's modulus $E=200 \mathrm{GPa}$, density $\rho=7800 \mathrm{~kg} / \mathrm{m}^{3}$, and Poisson's ratio $\nu=0.3$. The FE model is composed of 6,750 brick trilinear elements and has approximately 28,000 DOF. This FE model is used for all the numerical results in this paper, and the generation of the FE model as well as component mode synthesis were performed with the commercial code ANSYS. ${ }^{38}$

\section{Linear Free Response Analysis}

\section{III.A. Natural frequency variation due to variations in crack location and length}

For the FE model shown in Fig. 1, eigenvalue analysis was performed for various values of $l_{c} / l$ and $h_{c} / h$, and the results for the first 15 natural frequencies for two representative cases are shown in Fig. 2. First, Fig. 2 a shows the results where the crack length was fixed at $l_{c} / l=40 \%$, and the crack location was varied as $1.33 \leqslant h_{c} / h \leqslant 97.3 \%$. As can be seen, the changes in the natural frequencies due to the variation in $h_{c} / h$ are quite complicated, and multiple loci veerings and crossings are observed. For example in Fig. 2a, starting around $h_{c} / h=17 \%$, modes 10 and 11 approach each other, but rather than crossing they veer away near $h_{c} / h=19 \%$ with high curvature. Second, the crack location was fixed at $h / h_{c}=50 \%$, and the crack length 


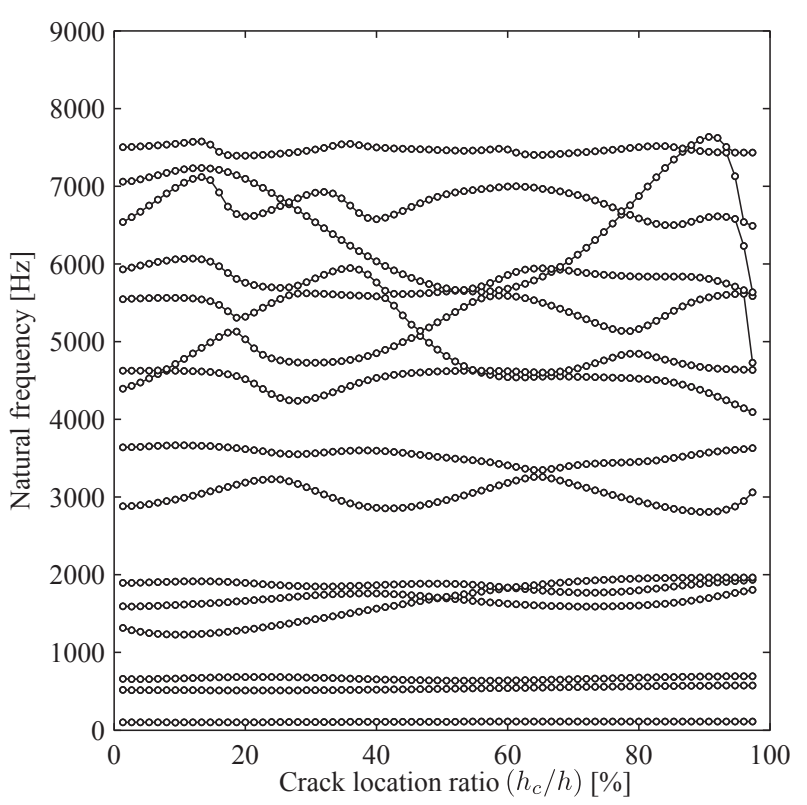

(a)

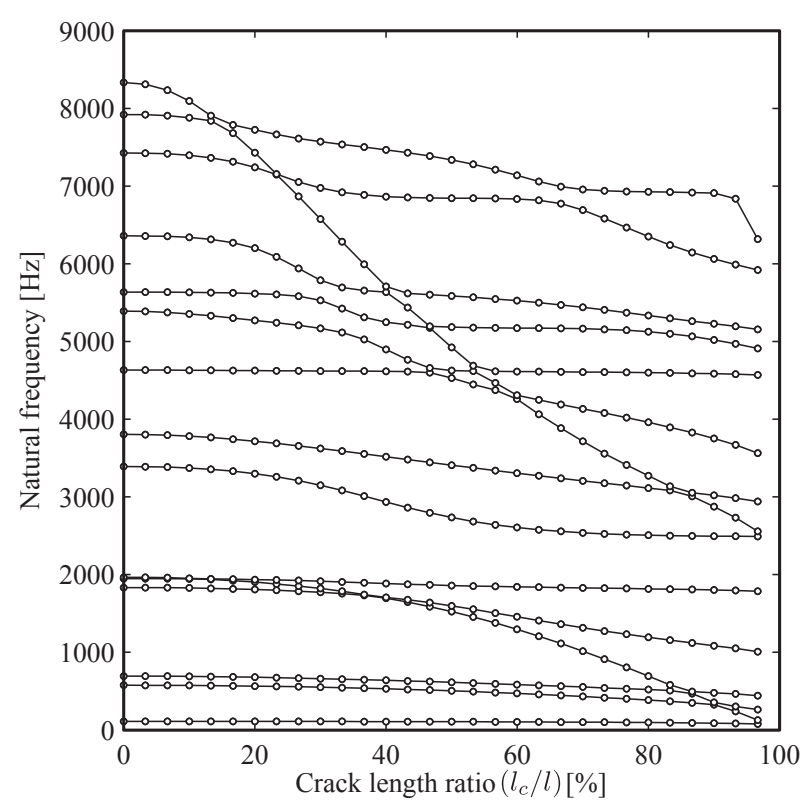

(b)

Figure 2. First 15 natural frequencies versus (a) crack location ratio $h_{c} / h$ for $l_{c} / l=0.40$, (b) crack length ratio $l_{c} / l$ for $h_{c} / h=0.50$

was varied as $0 \leqslant l_{c} / l \leqslant 96.7 \%$, the results of which are shown in Fig. $2 \mathrm{~b}$. The most notable distinction from the case in Fig. 2a is that the natural frequency variation due to crack length change is monotonic, i.e., as $l_{c} / l$ increases, all natural frequencies tend to decrease. Although the amount of frequency drop is dependent on the mode of interest, this is due to the fact that the stiffness of the plate monotonically decreases for all modes as the crack length increases.

\section{III.B. Mode shape variation due to variations in crack location and length}

In order to see the veering regions more closely, and to see the variations in the mode shapes, representative cases are shown in Figs. 3 and 4. Figure 3a shows the veering between the modes 10 and 11 for $l_{c} / l=40 \%$, where $1.33 \leqslant h_{c} / h \leqslant 40 \%$. An important characteristic of the loci veering is the mode shapes associated with the natural frequencies on each locus before veering are interchanged during the veering in a continuous manner. ${ }^{4}$ This is illustrated in Fig. 3a, which shows that mode shapes 10 and 11 become mixed and then appear to begin switching as the crack location ratio is increased through the veering region. On the other hand in Fig. 3b, the region for the mode shape switching between modes five and six is narrow, and it appears to be a loci crossing. This can be explained by considering that mode five (before switching) corresponds to the second out-of-plane bending mode whereas mode six (before switching) corresponds to the first in-plane bending mode, and there is little or no coupling between these modes due to their geometric dissimilarity. Fig. 4 shows another veering region due to crack length variation, for modes seven and eight with crack location $h_{c} / h=0.63$. For this case, both mode mixing and switching can be observed as the crack length is increased. In other words, the modes are coupled during the veering.

\section{Nonlinear Forced Response Analysis}

In the previous section, the interchanging of modes as well as mode coupling were observed in frequency veering and crossing regions. However, only natural frequencies of the linear system were considered. The nonlinearity due to contact of the crack surfaces was neglected. In this section, a method to calculate the 


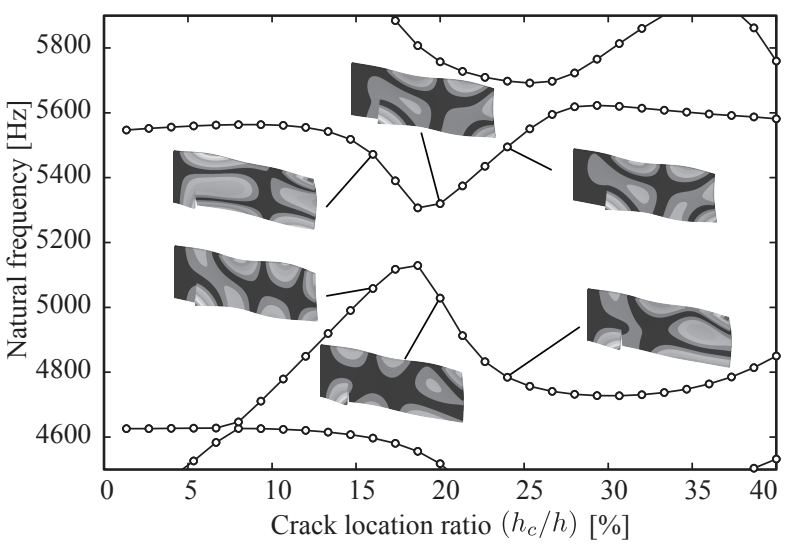

(a)

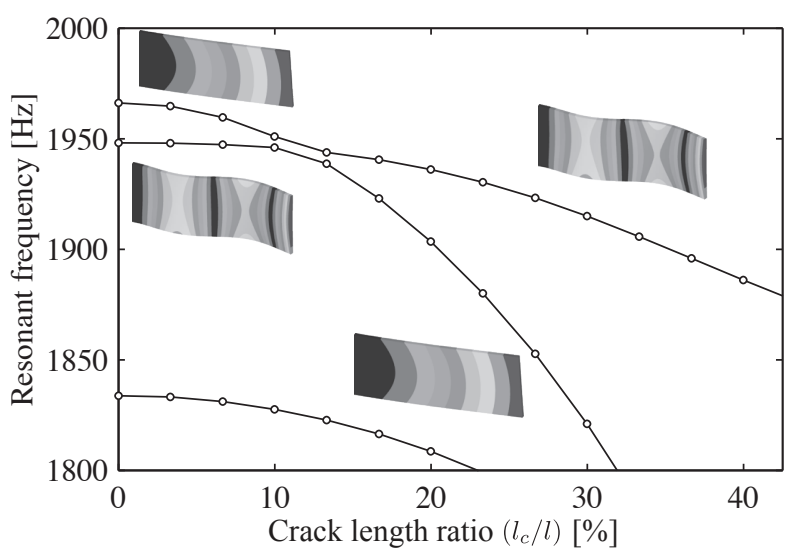

(b)

Figure 3. Magnified veeering/crossing regions and associated mode shapes: (a) 10th and 11th modes for $l_{c} / l=0.40$; (b) fifth and sixth modes for $h_{c} / h=0.50$.

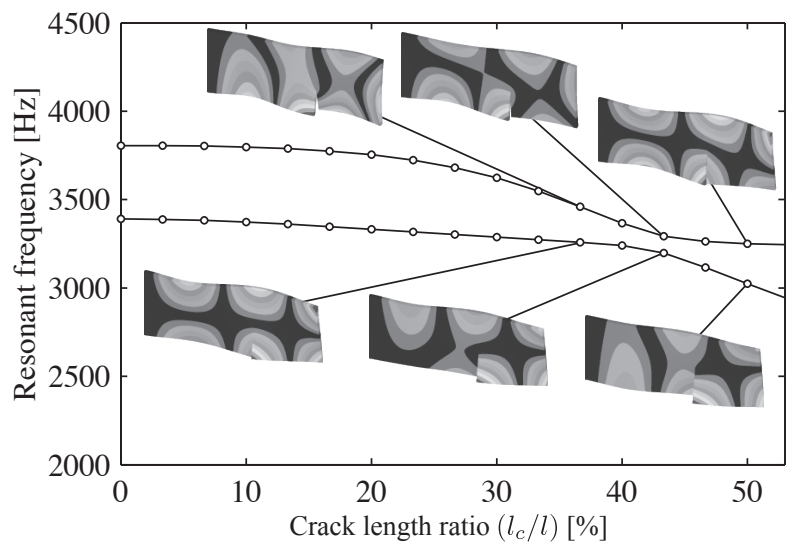

Figure 4. Magnified veering between modes seven and eight for $h_{c} / h=0.63$

nonlinear resonant frequencies of the cracked plate is described. The method is then applied to the calculation of nonlinear resonant frequencies in veering/crossing regions, and their characteristics are discussed.

\section{IV.A. Component Mode Synthesis}

In order to generate a reduced-order model, the plate is separated into two components (substructures) $\Omega_{1}$ and $\Omega_{2}$ along the crack path, as shown in Fig. 5, and a hybrid-interface method of component mode synthesis $^{39}$ is employed. This process is advantageous over the direct application of FE analysis because it provides improved computational efficiency while maintaining direct access to the dynamics of the cracksurface DOF. Furthermore, it has good accuracy relative to the original FE model over the frequency range of interest. The accessibility to the nodes on the crack surfaces is essential to the proper calculation of the boundary condition at the crack surfaces, which is modeled as contact/impact forces in the formulation described in IV.B. Namely, the dynamics of the FE degrees of freedom are projected onto constraint modes $\Psi_{c}$, inertia relief attachment modes $\boldsymbol{\Psi}_{a}$ (if rigid-body motion exists), and a truncated set of free-interface normal modes $\boldsymbol{\Phi}_{k}$. Interested readers may consult, e.g., Craig, ${ }^{40}$ for the detailed formulation of each mode set.

Let the displacement vector $\mathbf{u}$ be partitioned into boundary DOF, $\mathbf{u}_{b}$, and interior DOF $\mathbf{u}_{i}$. By denoting the inertia relief attachment coordinates and a truncated set of free-interface modal coordinates as $\mathbf{q}_{a}$ and 


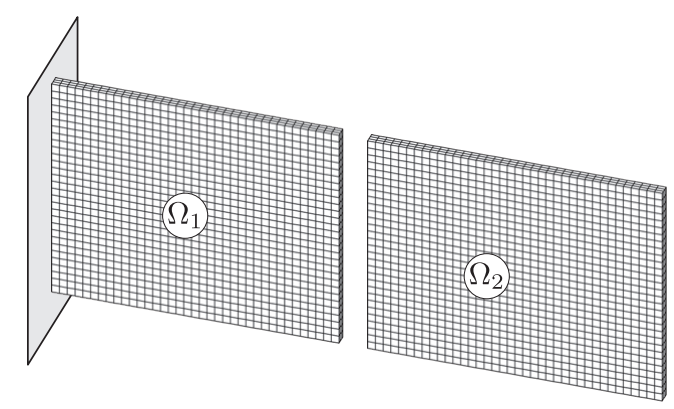

Figure 5. Plate divided into two substructures

$\mathbf{q}_{k}$, the linear projection is expressed as,

$$
\left[\begin{array}{c}
\mathbf{u}_{b} \\
\mathbf{u}_{i}
\end{array}\right]=\left(\begin{array}{ccc}
\mathbf{I} & \mathbf{0} & \mathbf{0} \\
\mathbf{\Psi}_{i c} & \hat{\mathbf{\Psi}}_{a} & \hat{\mathbf{\Psi}}_{k}
\end{array}\right)\left[\begin{array}{c}
\mathbf{u}_{b} \\
\mathbf{q}_{a} \\
\mathbf{q}_{k}
\end{array}\right]
$$

where $\hat{\boldsymbol{\Psi}}_{a}=\boldsymbol{\Psi}_{i a}-\boldsymbol{\Psi}_{i c} \boldsymbol{\Psi}_{b a}, \hat{\boldsymbol{\Psi}}_{k}=\boldsymbol{\Phi}_{i k}-\boldsymbol{\Psi}_{i c} \boldsymbol{\Phi}_{b k}$, I is the identity matrix, $\boldsymbol{\Psi}_{i c}$ is the boundary partition of $\boldsymbol{\Psi}_{c}, \boldsymbol{\Psi}_{i a}$ and $\boldsymbol{\Psi}_{b a}$ denote the interior and the boundary partitions of $\boldsymbol{\Psi}_{a}$, and $\boldsymbol{\Phi}_{i k}$ and $\boldsymbol{\Phi}_{b k}$ denote the interior and the boundary partitions of $\boldsymbol{\Phi}_{k}$. Denoting Eq. (2) with a compact notation, $\mathbf{u}=\boldsymbol{\Psi} \mathbf{q}$, the application of Eq. (2) to Eq. (1) yields a smaller number of equations, i.e.,

$$
\mathbf{M}^{\prime} \ddot{\mathbf{q}}+\mathbf{C}^{\prime} \dot{\mathbf{q}}+\mathbf{K}^{\prime} \mathbf{q}=\mathbf{b}^{\prime}+\mathbf{f}^{\prime}(\mathbf{q})
$$

where $\mathbf{M}^{\prime}=\Psi^{\mathrm{T}} \mathbf{M} \boldsymbol{\Psi}, \mathbf{C}^{\prime}=\boldsymbol{\Psi}^{\mathrm{T}} \mathbf{C} \boldsymbol{\Psi}, \mathbf{K}^{\prime}=\boldsymbol{\Psi}^{\mathrm{T}} \mathbf{K} \boldsymbol{\Psi}, \mathbf{b}^{\prime}=\boldsymbol{\Psi}^{\mathrm{T}} \mathbf{b}$, and $\mathbf{f}^{\prime}=\boldsymbol{\Psi}^{\mathrm{T}} \mathbf{f}$. The superscript "," is omitted for convenience in the subsequent formulations.

\section{IV.B. Hybrid frequency/time domain method}

For the calculation of steady-state response to harmonic excitation, an extension to the alternating frequency/timedomain method, ${ }^{41}$ which is based on the concept of the method of harmonic balance, ${ }^{42}$ is employed in this study. Because of its computational efficiency and accuracy, this type of method has been developed and applied to forced response problems for various nonlinear systems, such as friction damped systems ${ }^{43-46}$ and cracked shafts. ${ }^{47}$ In particular, the hybrid frequency/time-domain method developed by Poudou et al. ${ }^{48-50}$ and the authors ${ }^{51}$ is applied in this paper. Namely, the method assumes that the steady-state vibration response of $\mathbf{q}$ in Eq. (3), as well as the external force $\mathbf{b}$ and the nonlinear force due to intermittent contact $\mathbf{f}$ are approximated as truncated Fourier series with, i.e.,

$$
\begin{aligned}
& \mathbf{q}=\Re\left(\sum_{k=0}^{n_{h}}\left(\mathbf{Q}_{k}^{c}-\mathbf{j} \mathbf{Q}_{k}^{s}\right) \mathrm{e}^{\mathrm{j} k \omega t}\right) \\
& \mathbf{b}=\Re\left(\sum_{k=0}^{n_{h}}\left(\mathbf{B}_{k}^{c}-\mathrm{j} \mathbf{B}_{k}^{s}\right) \mathrm{e}^{\mathrm{j} k \omega t}\right) \\
& \mathbf{f}=\Re\left(\sum_{k=0}^{n_{h}}\left(\mathbf{F}_{k}^{c}-\mathbf{j} \mathbf{F}_{k}^{s}\right) \mathrm{e}^{\mathrm{j} k \omega t}\right)
\end{aligned}
$$

where $2 \pi / \omega$ is the fundamental frequency, $n_{h}$ is the number of non-zero harmonics and $\mathrm{j}=\sqrt{-1}$. Note that $\mathbf{Q}_{k}^{c}$ and $-\mathbf{Q}_{k}^{s}$ are the vectors of real and imaginary parts of $k$ th Fourier coefficients of $\mathbf{q}$, where superscripts 


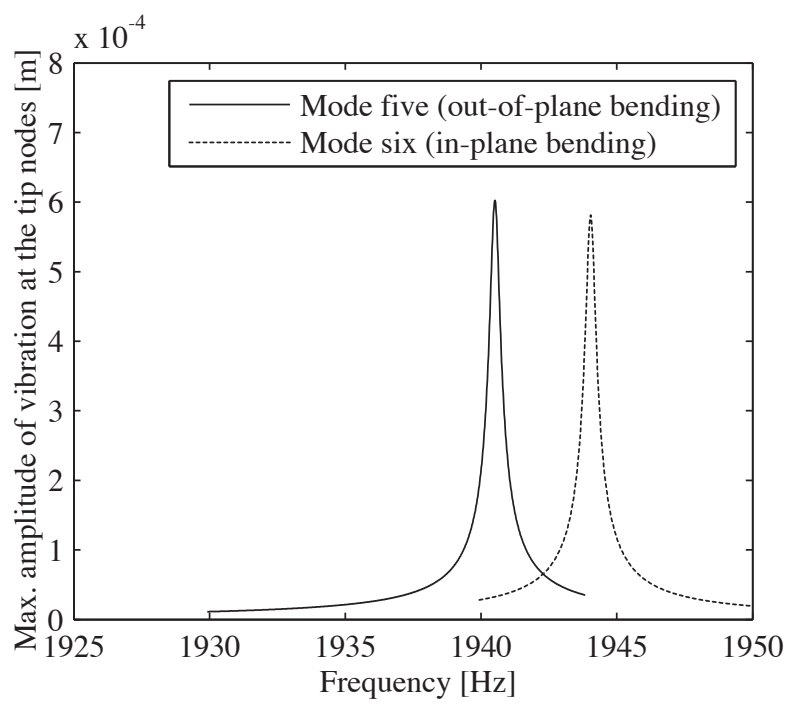

(a) $l_{c} / l=0.167$

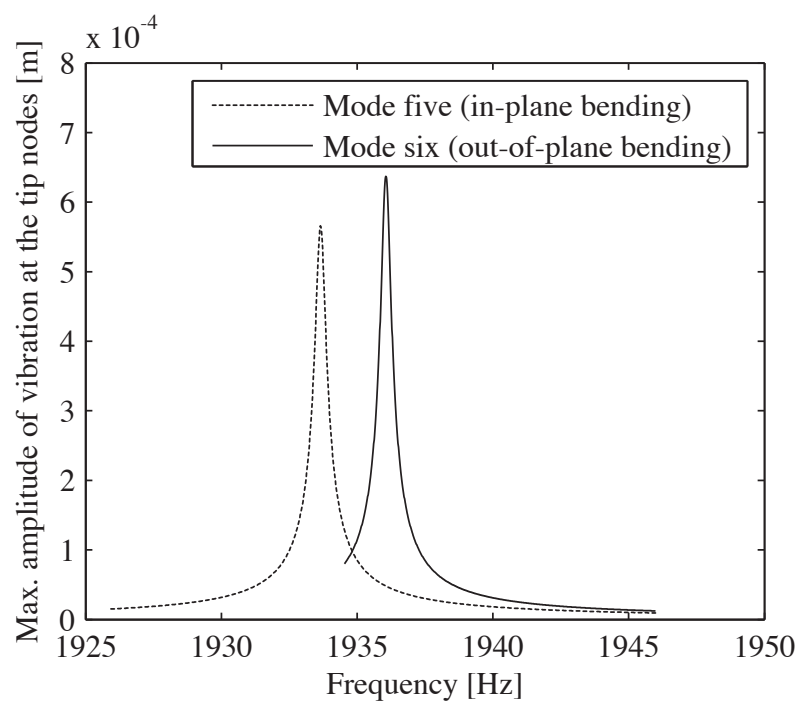

(b) $l_{c} / l=0.200$

Figure 6. Results of nonlinear harmonic response analysis for $h_{c} / h=0.5$

$c$ and $s$ denote cosine and sine components of the vibration respectively. The same notation is applied to $\mathbf{B}_{k}^{c}, \mathbf{B}_{k}^{s}, \mathbf{F}_{k}^{c}$, and $\mathbf{F}_{k}^{s}$. Substituting Eqs. (4) through (6) into Eq. (3) and considering the orthogonality of harmonic functions, it results in a nonlinear algebraic equation with respect to the Fourier coefficients for $k$ th harmonic number, i.e.,

$$
\boldsymbol{\Lambda}_{k} \mathbf{Q}_{k}=\mathbf{B}_{k}+\mathbf{F}_{k}(\mathbf{Q})
$$

where $\mathbf{Q}_{0}=\mathbf{Q}_{0}^{c}, \mathbf{B}_{0}=\mathbf{B}_{0}^{c}, \mathbf{F}_{0}=\mathbf{F}_{0}^{c}, \boldsymbol{\Lambda}_{0}=\mathbf{K}, \mathbf{Q}_{k}=\left[\left(\mathbf{Q}_{k}^{c}\right)^{\mathrm{T}},\left(\mathbf{Q}_{k}^{s}\right)^{\mathrm{T}}\right]^{\mathrm{T}}, \mathbf{B}_{k}=\left[\left(\mathbf{B}_{k}^{c}\right)^{\mathrm{T}},\left(\mathbf{B}_{k}^{s}\right)^{\mathrm{T}}\right]^{\mathrm{T}}$, $\mathbf{F}_{k}=\left[\left(\mathbf{F}_{k}^{c}\right)^{\mathrm{T}},\left(\mathbf{F}_{k}^{s}\right)^{\mathrm{T}}\right]^{\mathrm{T}}$, and

$$
\boldsymbol{\Lambda}_{k}=\left(\begin{array}{cc}
-(k \omega)^{2} \mathbf{M}+\mathbf{K} & (k \omega) \mathbf{C} \\
-(k \omega) \mathbf{C} & -(k \omega)^{2} \mathbf{M}+\mathbf{K}
\end{array}\right)
$$

for $k=1, \ldots, n_{h}$. Assembling Eq. (8) for all $k=0,1, \ldots, n_{h}$,

$$
\Lambda \mathbf{Q}=\mathbf{B}+\mathbf{F}(\mathbf{Q})
$$

where $\boldsymbol{\Lambda}$ is a pseudo-block diagonal matrix with $\Lambda_{k}$ on its diagonal blocks for $k=0, \ldots n_{h}, \mathbf{Q}=$ $\left[\mathbf{Q}_{0}^{\mathrm{T}}, \ldots, \mathbf{Q}_{n_{h}}^{\mathrm{T}}\right]^{\mathrm{T}}, \mathbf{B}=\left[\mathbf{B}_{0}^{\mathrm{T}}, \ldots, \mathbf{B}_{n_{h}}^{\mathrm{T}}\right]^{\mathrm{T}}$, and $\mathbf{F}=\left[\mathbf{F}_{0}^{\mathrm{T}}, \ldots, \mathbf{F}_{n_{h}}^{\mathrm{T}}\right]^{\mathrm{T}}$. Eq. (9) can then be solved with nonlinear algebraic equation solvers. For the numerical examples shown in this paper, the Hybrid Powell method ${ }^{52}$ was employed.

\section{IV.C. Results of forced response analysis}

In this subsection, the result of nonlinear forced response analysis for the cantilevered cracked plate is presented, with the methods described in IV.A and IV.B. The damping was chosen to be $\mathbf{C}=\alpha \mathbf{M}+\beta \mathbf{K}$ where $\alpha=1.22$ and $\beta=8.16 \times 10^{-9}$, which result in damping that is approximately equivalent to modal (structural) damping ratio $\zeta=1.00 \times 10^{-4}\left(\gamma=2.00 \times 10^{-4}\right)$ within the frequency range of $1900 \leqslant$ $f \leqslant 2000 \mathrm{~Hz}$. Vectors of harmonic forcing, the resultant of which is equal to $1 \mathrm{~N}$, is applied to the nodes on the tip face of the plate to excite the modes of interest. The number of harmonics was chosen as $n_{h}=9$, which showed convergence in the frequency response. Representative results are shown in Fig. 6 where 


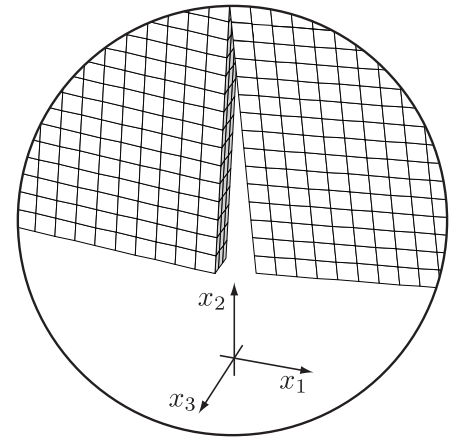

(a)

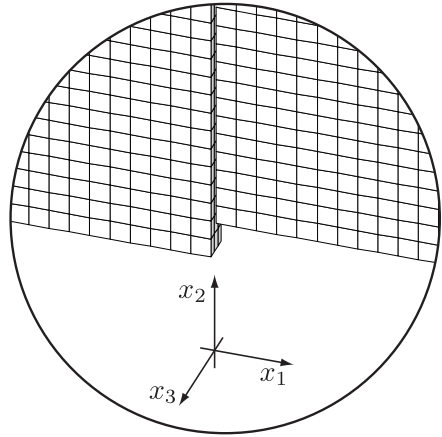

(b)

Figure 7. Constraints for bilinear frequency calculation: (a) Open (no constraints); (b) Closed (sliding).

$h_{c} / h=0.5, l_{c} / l=0.167$ for Fig. 6 a, and $l_{c} / l=0.2$ for Fig. 6b. Fig. 6a shows the resonant peaks corresponding to modes five and six, which correspond to the third out-of-plane bending and the first inplane bending modes, respectively, whereas the order of the modes is interchanged in Fig. $6 \mathrm{~b}$.

\section{Bilinear Frequency Approximation}

\section{V.A. Formulation}

As an alternative way of predicting the nonlinear vibration frequencies, the bilinear frequency approximation is extended for the analysis of three-dimensional cracked structures, and an analysis framework based on reduced-order modeling as well as prediction of mode switching during the veering regions is proposed in this section. The resonant peaks predicted by the forced response to harmonic excitation is then compared with those calculated by the bilinear frequency approximations.

The bilinear frequency was originally introduced as the effective vibration frequency of a piecewise linear, single-DOF system and defined as, ${ }^{28}$

$$
\omega_{b}=\frac{2 \omega_{1} \omega_{2}}{\omega_{1}+\omega_{2}}
$$

where $\omega_{b}$ is the bilinear frequency, $\omega_{1}$ is the natural frequency of one of the linear systems associated with the piecewise linear system, and $\omega_{2}$ is that of the other linear system of the piecewise linear system. This expression is the exact solution, for the frequency of free oscillation of the piecewise linear single-DOF oscillator with vanishing clearance/gap at the equilibrium. The application of Eq. (10) to a multi-DOF piecewise linear system is rather straightforward if there is only one pair of linear systems. However in the cases of cracked plates formulated with multiple DOF on crack surfaces, it involves multiple piecewise linear systems, or a conewise linear systems. ${ }^{34}$ Hence an assumption has to be made such that the cracked system has only two linear systems corresponding to two states, i.e., the crack is open or closed. These are designated as states 1 and 2, respectively, in the following formulation. The definition of the states 1 and 2 is a natural extension to that proposed by Chati et al. ${ }^{32}$ which was applied to the analysis of in-plane bending vibrations of a cracked beam. Namely, with the assumption of the open state, there is no connection between the nodes on one crack surface and the nodes on the other surface (Fig. 7a), allowing the inter-penetration of the crack surfaces. On the other hand with the closed state, the relative DOF along the direction that is perpendicular to the crack surfaces are fixed to be zero, whereas the other two DOF of each node are allowed to move freely in the plane tangent to the constrained direction (Fig. 7b). In other words, the crack surfaces are allowed to slide with respect to each other, which is consistent with the assumption employed in the 
formulation in IV.B. Associated mathematical formulation is given as follows.

For a given crack length, eigenvalues of Eq. (1) for undamped case with open crack assumption are obtained as

$$
\mathbf{K} \phi=\omega_{1}^{2} \mathbf{M} \phi
$$

where $\phi$ is the eigenvector and $\omega_{1}^{2}$ is the associated eigenvalue. On the other hand, the eigenvalues and eigenvectors for the other case, namely the case with allowing sliding of crack surfaces, are obtained by imposing appropriate constraints on Eq. (11) as follows. Let $A$ and $B$ denote the crack surfaces facing each other, by assuming that the amplitude of vibration is much smaller than the finite element mesh size on the crack surfaces, it is possible to identify the finite element nodes that may be in contact during the vibration cycle. Hence such pairs of nodes are numbered and a set $\mathcal{C}_{c p}$ is defined where all numbers that denote the contact pairs are included. Defining $g_{n}$ as the gap between the nodes on the surfaces $A$ and $B$ for the $n$th contact pair, the constraints to be imposed on the nodes of $n$th contact pair are expressed as

$$
g_{n}=\left(u_{n}\right)_{A}-\left(u_{n}\right)_{B}=0, \quad n \in \mathcal{C}_{c p}
$$

where $\left(u_{n}\right)_{A}$ and $\left(u_{n}\right)_{B}$ denote the displacements of the nodes on the surface $A$ and $B$, projected onto the normal direction pointing outward from the surface $A$ or $B$. It is noted that appropriate coordinate transformation must be applied to the displacement vector based on the normal vector at each node, in order to correctly calculate $g_{n}$. It should also be noted that the motion of the nodes in tangential plane that is perpendicular to the normal direction, is not constrained at all by Eq. (12), i.e., the nodes are free to slide with each other on the tangential plane. This also indicates that the crack surfaces are assumed to be frictionless, which is widely-employed assumption for the vibration problem of cracked beams and plates. Applying the constraints Eq. (12) to the eigenvalue problem Eq. (11), a constrained eigenvalue problem is obtained as

$$
\left[\begin{array}{cc}
\mathbf{K} & \mathbf{N}^{\mathrm{T}} \\
\mathbf{N} & 0
\end{array}\right]\left[\begin{array}{l}
\boldsymbol{\phi} \\
\boldsymbol{\lambda}
\end{array}\right]=\omega_{2}^{2}\left[\begin{array}{cc}
\mathbf{M} & \mathbf{0} \\
\mathbf{0} & \mathbf{0}
\end{array}\right]\left[\begin{array}{l}
\boldsymbol{\phi} \\
\boldsymbol{\lambda}
\end{array}\right]
$$

where $\mathbf{N}$ is the matrix of coefficients that are associated with Eq. (12) and the appropriate transformation matrix, and $\lambda$ is the vector of Lagrange multipliers of size $\left|\mathcal{C}_{c p}\right|$. One method to solve this indefinite eigenvalue problem is to use an eigenvalue solver for indefinite systems. Another method is to first eliminate the redundant equations due to the constraint equations Eq. (12), and the resulting positive definite eigenvalue problem is then solved by an eigenvalue solver for definite systems. It should be noted that this methodology can easily be incorporated with the reduced-order modeling framework described in IV.A as the motion of the nodes on the crack surfaces in the three-dimensional space can be captured with the reduced-order model.

With the eigenvalue problems Eqs. (12) and (13), the $i$ th bilinear resonant frequency $\omega_{b i}$ of the cracked plate is approximated based on Eq. (10):

$$
\omega_{b i}=\frac{2 \omega_{1 i} \omega_{2 i}}{\omega_{1 i}+\omega_{2 i}}
$$

where $\omega_{1 i}$ and $\omega_{2 i}$ denote the frequencies of the $i$ th mode of the states 1 and 2 . It is emphasized that the index $i$ does not denote the index of eigenvalues, but it denotes the index of the eigenvectors of the non-cracked plate. Namely, the eigenvectors of the non-cracked plate are indexed based on their natural frequencies, i.e., for non-cracked plate, the eigenvalues are ordered as $\omega_{1} \leqslant \omega_{2} \leqslant \cdots \leqslant \omega_{N-1} \leqslant \omega_{N}$ where $N$ is the size of the non-cracked plate model, and corresponding eigenvectors are labeled as $\left[\phi_{1}, \phi_{2}, \ldots, \phi_{N-1}, \phi_{N}\right]$. The bilinear frequency $\omega_{b i}$ for a given crack length is calculated by using the natural frequencies of the corresponding $i$ th mode of the states 1 and 2 .

The advantage of this method is that the frequency of the nonlinear response is obtained without calculating the associated mode shapes, thus it only involves eigenvalue extraction of two linear systems. However, 


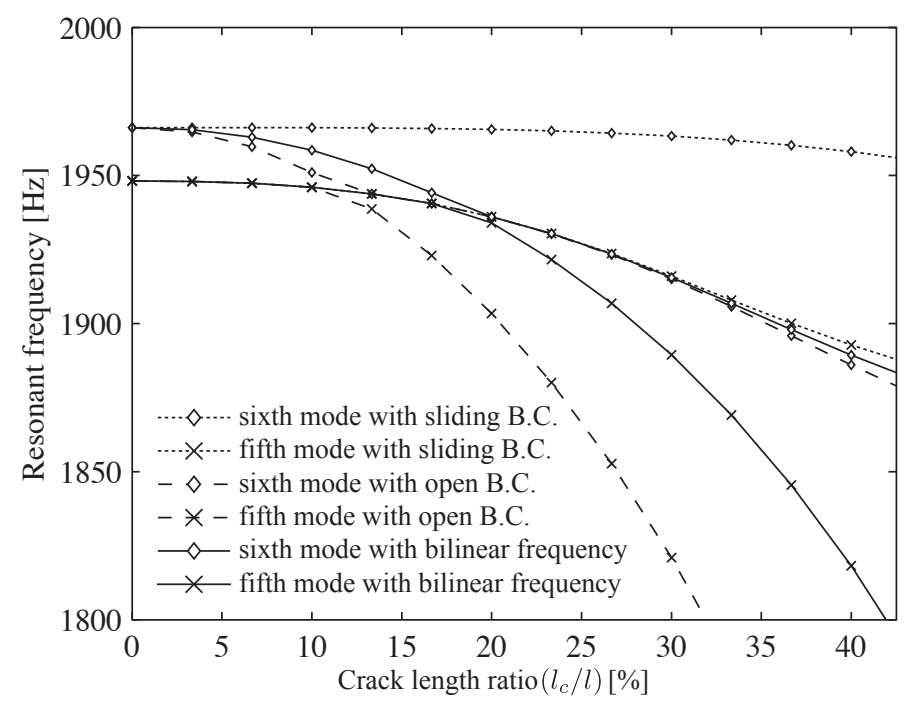

Figure 8. The fifth and sixth natural frequencies of the system where $h_{c} / h=0.50$ with sliding and open B.C.s, and bilinear frequencies

as mentioned, this method is known to be accurate for systems with a relatively short crack. In addition, a drawback of this method is that the choice of proper pairs of $\omega_{1 i}$ and $\omega_{2 i}$ is not apparent with the presence of a veering or crossing, because the mode shapes associated with the natural frequencies switch their orders. A way to overcome the latter problem is to track each mode by observing the correlation between the modes during the variation of crack length or crack location. In this paper, the modal assurance criterion ${ }^{53}$ (MAC) is employed as the measure of correlation.

Denoting the crack length as $p\left(=l_{c}\right)$, it is noted that $\mathbf{N}$ and $\boldsymbol{\lambda}$ are dependent on $p$. That is, $\mathbf{N}=\mathbf{N}(p)$ and $\boldsymbol{\lambda}=\boldsymbol{\lambda}(p)$. The eigenvector is also dependent on $p, \boldsymbol{\phi}=\boldsymbol{\phi}(p)$, and the correlation between the $i$ th mode shape of the system with $p=p_{0}$ and the $j$ th mode shape with the perturbed crack length $p=p_{0}+\Delta p$ can be characterized by

$$
M A C_{i j}^{k}=\frac{\left|\boldsymbol{\phi}_{i}^{k}\left(p_{0}\right)^{\mathrm{T}} \boldsymbol{\phi}_{j}^{k}\left(p_{0}+\Delta p\right)\right|^{2}}{\left\|\boldsymbol{\phi}_{i}^{k}\left(p_{0}\right)\right\|^{2}\left\|\boldsymbol{\phi}_{j}^{k}\left(p_{0}+\Delta p\right)\right\|^{2}}, \quad k=1,2
$$

where $\phi$ is the eigenvector of the system defined by Eq. (13), the subscripts $i$ and $j$ denote the indices for modes, the superscript $k$ indicates the state, and $M A C_{i j}^{k}$ takes the value between 0 and 1 , which respectively correspond to no correlation, and consistent correlation between $\phi_{i}\left(p_{0}\right)$ and $\phi_{j}\left(p_{0}+\Delta p\right)$. Namely, the $i$ th eigenvector is tracked based on the value of $M A C$ throughout the variation of the crack length $(p)$, such that the correct natural frequencies for the $i$ th eigenvector in Eq. (14) can be calculated.

In order to better clarify the behavior of the natural frequencies of the system with open and sliding boundary conditions, as well as the bilinear frequencies, the above mentioned analysis framework was applied to the reduced-order model of the cracked plate with $h_{c} / h=0.50$. As an example, the veering region between the fifth and sixth modes are shown in Fig. 8. As was shown in III.B, the modes of interest are the in-plane and out-of-plane bending modes. In Fig. 8, two significant insights into the behavior of the frequencies are shown. The first is that the existence and location vary between the cases with open and sliding boundary conditions, and bilinear frequency. For the case with sliding boundary condition, the veering between fifth and sixth modes does not exist. On the other hand for the open boundary condition case, the loci of fifth and sixth modes approach and veer away where $10 \leqslant l_{c} / l \leqslant 15 \%$. Therefore the bilinear frequency also has the veering region due to that for the open boundary condition, but slightly shifted toward larger crack length ratio because of the absence of the veering for the sliding boundary condition case. The second is that the bilinear frequency is always bounded by the frequencies corresponding to the cases with sliding and open boundary conditions, which are respectively the upper and lower bounds. 
Furthermore, it is noted that the width between the upper and lower bounds indicates the strength of the effect of contact nonlinearity on the resonant frequency. For instance, for the fifth bilinear frequency that corresponds to the in-plane bending mode, the width between the bounds is much larger than that for the sixth bilinear frequency, which corresponds to the out-of-plane bending mode. This is due to the fact that the motion of the in-plane bending mode is greatly influenced by the existence of the contact force at the crack surfaces, whereas the out-of-plane bending modes is not so much affected by the contact force considering that the motion of the crack surfaces is almost perpendicular to the crack surfaces.

\section{V.B. Comparison with the results of forced response analysis}

Using the bilinear frequency approximation described above, the nonlinear vibration frequencies of the cracked plate are calculated, and they are compared with those obtained by the HFT method. It is noted that the comparison between the resonant frequencies obtained by forced response analysis, and the bilinear frequencies, namely the vibration frequencies of unforced system, has been made based on the assumption that the resonant frequencies reside in the vicinity of the frequencies associated with the nonlinear normal modes ${ }^{54}$ Furthermore, the resonant frequencies are assumed to be independent of the amplitude of forcing, based on the fact that the resonant frequencies of piecewise linear systems with the vanishing gap at the equilibrium are not dependent on vibration amplitude. ${ }^{28,51}$

Three representative veering regions are considered, which are the cases where (a) the interaction between the loci is weak and the corresponding modes are: (1) in-plane and out-of-plane bending modes, and (2) both out-of-plane bending modes, and (b) the interaction between the loci is strong and veering occurs in a continuous way and the associated modes are both out-of-plane bending modes.

First, the veering between an in-plane bending mode and an out-of-plane bending mode is considered, using the modes five and six, for $h_{c} / h=0.50$, as shown in Fig. 3b. The results of forced response analysis as well as the calculation based on bilinear frequency assumption are shown in Fig. 9a. As can be seen, the order-switching of modes can be observed even for this nonlinear system. The most notable distinction from the linear assumption, i.e., Fig. 3b, is that the veering occurs with longer crack length, i.e., around 20\% whereas it occurs around $10 \%$ in Fig. 3b. This is due to the stiffening effect because of the contact/impact of crack surfaces during the vibration cycle, which represents the dynamics of the cracked plates appropriately. Regarding the bilinear frequency approximation, a notable result has been observed: the bilinear frequency assumption predicts the resonant frequency calculated by HFT method quite well even for relatively large crack length ratio $\left(l_{c} / l \leqslant 40 \%\right)$.

Second, the veering between two out-of-plane bending modes is considered, using the modes nine and ten for $h_{c} / h=0.60$, and the calculation results are shown in Fig. 9b. This result also shows that bilinear frequency approximates the resonant frequencies quite well for the case of veering between out-of-plane bending modes, with relatively large crack length. Even though the effect of nonlinearity on the vibration frequency is smaller than that on the in-plane bending modes, as it does not involve much contact/impact between crack surfaces, this clearly indicates that the bilinear frequency approximation can also be used for the prediction of nonlinear vibration frequencies of out-of-plane bending modes.

Third, the veering between the torsion and out-of-plane bending modes are examined, using the modes seven and eight for $h_{c} / h=0.63$ and results are shown in Fig. 9c. This veering region features a switching of modes in a continuous way, or in other words, the mode shapes gradually change as the crack length is varied. This result shows that the bilinear frequency approximation predicts the nonlinear vibration frequency quite well even for the modes that exhibit complicated geometry due to coupling between modes. Moreover, the results show that the approximation is accurate even for large cracks. 


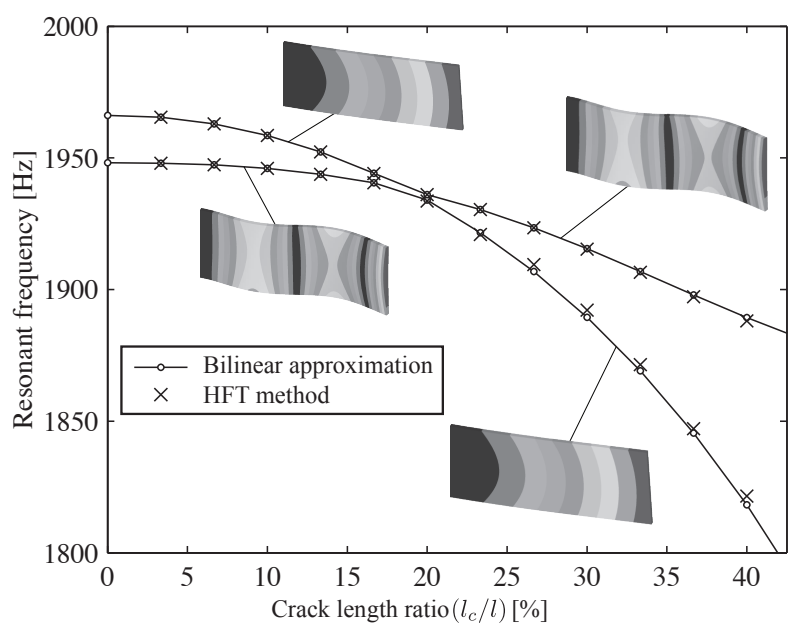

(a) $h_{c} / h=0.50$

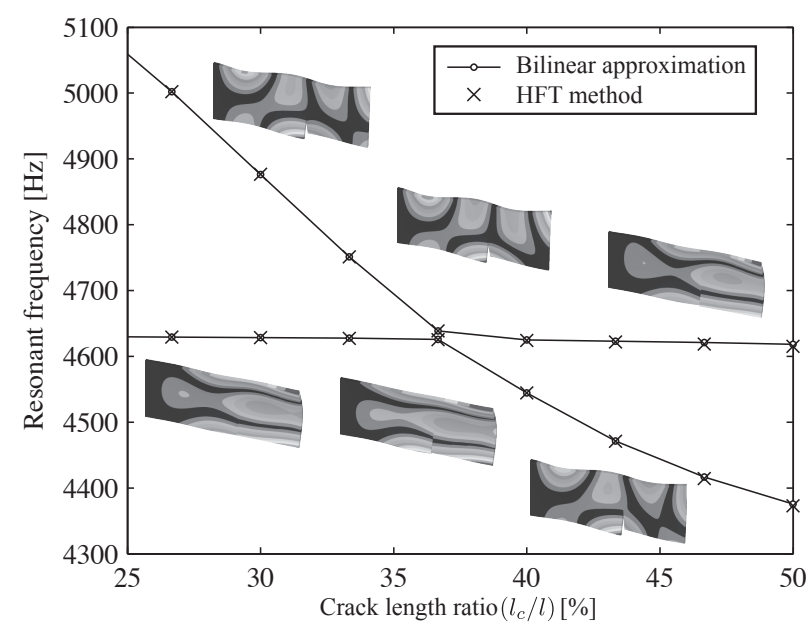

(b) $h_{c} / h=0.60$

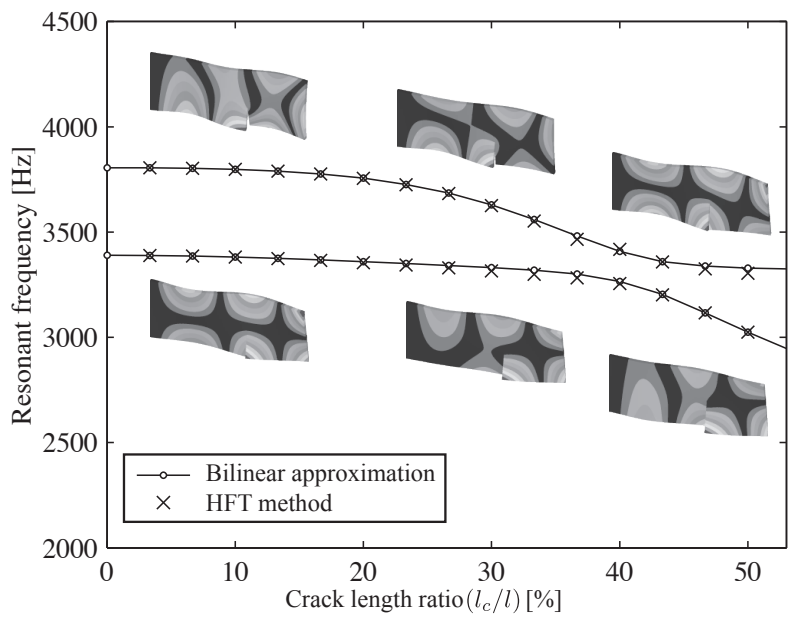

(c) $h_{c} / h=0.63$

Figure 9. Comparison between bilinear frequency assumption and HFT method, and corresponding mode shapes with open B.C.

\section{Conclusions}

In this paper, the linear and nonlinear vibration response of a cracked cantilevered rectangular plate has been investigated. In particular, the veering phenomena for the natural frequencies of the cracked plate were investigated. It was observed that veerings appear in plots of natural frequencies versus crack length or crack location ratio. It was shown that a wider veering region entails continuous interchanging between the modes, whereas a smaller veering (or crossing) region shows fast mode switching. Then, the nonlinear vibration response of the cracked plate due to contact of the crack surfaces was considered. A hybrid frequency/time-domain (HFT) method was applied to the calculation of nonlinear resonant frequencies in representative veering/crossing regions. It was shown that the characteristics of veerings/crossings are affected to some extent by the nonlinearity induced by the crack closing effect, although in general they are similar to those of the linear counterparts. Furthermore, an alternative method for estimating the nonlinear resonant frequencies was introduced by extending the bilinear frequency approximation. It was observed that the numerical results from the bilinear frequency approximation matched very well with those obtained by the HFT method, even for relatively large crack lengths. 


\section{References}

${ }^{1}$ Dimarogonas, A. D., "Vibration of Cracked Structures: A State of the Art Review," Engineering Fracture Mechanics, Vol. 55, No. 5, 1996, pp. 831-857.

${ }^{2}$ Kuttler, J. R. and Sigillito, V. G., “On Curve Veering,” Journal of Sound and Vibration, Vol. 75, No. 4, 1981, pp. 585-588.

${ }^{3}$ Leissa, A. W., "On a Curve Veering Aberration,” Zeitschrift für Angewandte Mathematik und Physik (ZAMP), Vol. 25, No. 1, 1974, pp. 99-111.

${ }^{4}$ Perkins, N. C. and Mote, C. D., "Comments on Curve Veering in Eigenvalue Problems," Journal of Sound and Vibration, Vol. 106, No. 3, 1986, pp. 451-463.

${ }^{5}$ Pierre, C. and Dowell, E. H., "Localization of Vibrations by Structural Irregularity," Journal of Sound and Vibration, Vol. 114, No. 3, 1987, pp. 549-564.

${ }^{6}$ Pierre, C., "Mode Localization and Eigenvalue Loci Veering Phenomena in Disordered Structures," Journal of Sound and Vibration, Vol. 126, No. 3, 1988, pp. 485-502.

${ }^{7}$ Triantafyllou, M. S. and Triantafyllou, G. S., "Frequency Coalescence and Mode Localization Phenomena: A Geometric Theory," Journal of Sound and Vibration, Vol. 150, No. 3, 1991, pp. 485-500.

${ }^{8}$ Vidoli, S. and Vestroni, F., "Veering Phenomena in Systems with Gyroscopic Coupling," Journal of Applied Mechanics, Vol. 72, No. 5, 2005, pp. 641-647.

${ }^{9}$ Challamel, N., Lanos, C., and Casandjian, C., "Localization in the Buckling or in the Vibration of a Two-Span Weakened Column,” Engineering Structures, Vol. 28, No. 5, 2006, pp. 776-782.

${ }^{10}$ Lepidi, M., Gattulli, V., and Vestroni, F., "Static and Dynamic Response of Elastic Suspended Cables with Damage," International Journal of Solids and Structures, Vol. 44, No. 25-26, 2007, pp. 8194-8212.

${ }^{11}$ Lynn, P. P. and Kumbasar, N., "Free Vibrations of Thin Rectangular Plates Having Narrow Cracks with Simply Supported Edges," Development in Mechanics, Vol. 4, 1967, pp. 911-928.

${ }^{12}$ Petyt, M., "The Vibration Characteristics of a Tensioned Plate Containing a Fatigue Crack," Journal of Sound and Vibration, Vol. 8, No. 3, 1968, pp. 377-389.

${ }^{13}$ Stahl, B. and Keer, L. M., "Vibration and Stability of Cracked Rectangular Plates," International Journal of Solids and Structures, Vol. 8, No. 1, 1972, pp. 69-91.

${ }^{14}$ Hirano, Y. and Okazaki, K., "Vibration of Cracked Rectangular-Plates," Bulletin of the JSME-Japan Society of Mechanical Engineers, Vol. 23, 1980, pp. 732-740.

${ }^{15}$ Solecki, R., "Bending Vibration of a Simply Supported Rectangular Plate with a Crack Parallel to One Edge," Engineering Fracture Mechanics, Vol. 18, No. 6, 1983, pp. 1111-1118.

${ }^{16}$ Yuan, J. and Dickinson, S. M., "The Flexural Vibration of Rectangular Plate Systems Approached by Using Artificial Springs in the Rayleigh-Ritz Method," Journal of Sound and Vibration, Vol. 159, No. 1, 1992, pp. 39-55.

${ }^{17}$ Liew, K. M., Hung, K. C., and Lim, M. K., “A Solution Method for Analysis of Cracked Plates Under Vibration,” Engineering Fracture Mechanics, Vol. 48, No. 3, 1994, pp. 393-404.

${ }^{18}$ Ma, C. C. and Huang, C. H., "Experimental and Numerical Analysis of Vibrating Cracked Plates at Resonant Frequencies," Experimental Mechanics, Vol. 41, No. 1, 2001, pp. 8-18.

${ }^{19}$ Christides, S. and Barr, A. D. S., "One-Dimensional Theory of Cracked Bernoulli-Euler Beams," International Journal of Mechanical Sciences, Vol. 26, No. 11-12, 1984, pp. 639-648.

${ }^{20}$ Shen, M. H. H. and Pierre, C., "Natural Modes of Bernoulli-Euler Beams with Symmetric Cracks," Journal of Sound and Vibration, Vol. 138, No. 1, 1990, pp. 115-134.

${ }^{21}$ Shen, M. H. H. and Pierre, C., "Free Vibrations of Beams with a Single-Edge Crack," Journal of Sound and Vibration, Vol. 170, No. 2, 1994, pp. 237-259.

${ }^{22}$ Chondros, T. G., Dimarogonas, A. D., and Yao, J., “A Continuous Cracked Beam Vibration Theory,” Journal of Sound and Vibration, Vol. 215, No. 1, 1998, pp. 17-34.

${ }^{23}$ Gudmundson, P., "The Dynamic Behavior of Slender Structures with Cross-Sectional Cracks," Journal of the Mechanics and Physics of Solids, Vol. 31, 1983, pp. 329-345.

${ }^{24}$ Pugno, N., Surace, C., and Ruotolo, R., "Evaluation of the Non-linear Dynamic Response to Harmonic Excitation of a Beam with Several Breathing Cracks," Journal of Sound and Vibration, Vol. 235, No. 5, 2000, pp. 749-762.

${ }^{25}$ Bovsunovsky, A. P. and Surace, C., "Consideration Regarding Superharmonic Vibrations of a Cracked Beam and the Variation in Damping Caused by the Presence of the Crack," Journal of Sound and Vibration, Vol. 288, 2005, pp. 865-886.

${ }^{26}$ Carpinteri, A. and Pugno, N., "Towards Chaos in Vibrating Damaged Structures-Part I: Theory and Period Doubling Cascade," Journal of Applied Mechanics, Vol. 72, No. 4, 2005, pp. 511-518.

${ }^{27}$ Andreaus, U., Casini, P., and Vestroni, F., "Non-linear Dynamics of a Cracked Cantilever Beam under Harmonic Excitation," International Journal of Non-Linear Mechanics, Vol. 42, 2007, pp. 566-575.

${ }^{28}$ Shaw, S. W. and Holmes, P. J., "A Periodically Forced Piecewise Linear Oscillator," Journal of Sound and Vibration, Vol. 90 , No. 1, 1983, pp. 129-155.

14 of 15 
${ }^{29}$ Butcher, E. A., "Clearance Effects on Bilinear Normal Mode Frequencies," Journal of Sound and Vibration, Vol. 224, 1999, pp. 305-328.

${ }^{30}$ Shen, M. H. H. and Chu, Y. C., "Vibrations of Beams with a Fatigue Crack," Computers and Structures, Vol. 45, 1992, pp. 79-93.

${ }^{31}$ Chondros, T. G., Dimarogonas, A. D., and Yao, J., "Vibration of a Beam with a Breathing Crack," Journal of Sound and Vibration, Vol. 239, Jan 2001, pp. 57-67.

${ }^{32}$ Chati, M., Rand, R., and Mukherjee, S., "Modal Analysis of a Cracked Beam," Journal of Sound and Vibration, Vol. 207, Oct 1997, pp. 249-270.

${ }^{33}$ Kisa, M. and Brandon, J., "The Effects of Closure of Cracks on the Dynamics of a Cracked Cantilever Beam," Journal of Sound and Vibration, Vol. 238, Nov 2000, pp. 1-18.

${ }^{34}$ Zuo, L. and Curnier, A., "Nonlinear Real and Complex-Modes of Conewise Linear-Systems," Journal of Sound and Vibration, Vol. 174, Jul 1994, pp. 289-313.

${ }^{35}$ Jiang, D., Pierre, C., and Shaw, S. W., "Large-Amplitude Non-Linear Normal Modes of Piecewise Linear Systems," Journal of Sound and Vibration, Vol. 272, 2004, pp. 869-891.

${ }^{36}$ Chen, S. L. and Shaw, S. W., "Normal Modes for Piecewise Linear Vibratory Systems," Nonlinear Dynamics, Vol. 10, Jun 1996, pp. 135-164.

${ }^{37}$ Lacarbonara, W., Arafat, H. N., and Nayfeh, A. H., "Non-Linear Interactions in Imperfect Beams at Veering," International Journal of Non-Linear Mechanics, Vol. 40, No. 7, 2005, pp. 987-1003.

${ }^{38}$ ANSYS, Inc., Pittsburgh, PA, ANSYS Release 10.0 Documentation, 2005.

${ }^{39}$ Herting, D. N., "A General purpose, Multi-Stage, Component Modal Synthesis Method," Finite Elements in Analysis and Design, Vol. 1, No. 2, 1985, pp. 153-164.

${ }^{40}$ Craig, R. R., Structural Dynamics: An Introduction to Computer Methods, John Wiley \& Sons, New York, 1981.

${ }^{41}$ Cameron, T. and Griffin, J., "An Alternating Frequency/Time Domain Method for Calculating the Steady-State Response of Nonlinear Dynamic Systems," Journal of Applied Mechanics, Vol. 56, 1989, pp. 149-154.

${ }^{42}$ Nayfeh, A. and Mook, D., Nonlinear Oscillations, John Wiley \& Sons, 1979.

${ }^{43}$ Pierre, C., Ferri, A. A., and Dowell, E. H., "Multi-Harmonic Analysis of Dry Friction Damped Systems Using an Incremental Harmonic Balance Method," Journal of Applied Mechanics, Vol. 52, 1985, pp. 958-964.

${ }^{44}$ Nacivet, S., Pierre, C., Thouverez, F., and Jezequel, L., "A Dynamic Lagrangian Frequency-Time Method for the Vibration of Dry-Friction-Damped Systems," Journal of Sound and Vibration, Vol. 265, Jul 2003, pp. 201-219.

${ }^{45}$ Petrov, E. P. and Ewins, D. J., "Advanced Modeling of Underplatform Friction Dampers for Analysis of Bladed Disk Vibration," Journal of Turbomachinery, Vol. 129, No. 1, 2007, pp. 143-150.

${ }^{46}$ Laxalde, D., Thouverez, F., Sinou, J. J., and Lombard, J. P., "Qualitative Analysis of Forced Response of Blisks with Friction Ring Dampers," European Journal of Mechanics - A/Solids, Vol. 26, No. 4, 2007, pp. 676-687.

${ }^{47}$ Sinou, J. J. and Lees, A. W., “A Non-Linear Study of a Cracked Rotor,” European Journal of Mechanics A/Solids, Vol. 26, Jan 2007, pp. 152-170.

${ }^{48}$ Poudou, O., Pierre, C., and Reisser, B., "A New Hybrid Frequency-Time Domain Method for the Forced Vibration of Elastic Structures With Friction and Intermittent Contact," Proceedings of the 10th International Symposium on Transport Phenomena and Dynamics of Rotating Machinery, paper ISROMAC10-2004-068, Honolulu, Hawaii, March 2004.

${ }^{49}$ Poudou, O. and Pierre, C., "A New Method for the Analysis of the Nonlinear Dynamics of Structures With Cracks," Proceedings of NOVEM 2005, Saint-Raphaël, France, April 2005.

${ }^{50}$ Poudou, O., Modeling and Analysis of the Dynamics of Dry-Friction-Damped Structural Systems, Ph.D. Dissertation, The University of Michigan, 2007.

${ }^{51}$ Saito, A., Castanier, M. P., and Pierre, C., "Efficient Nonlinear Vibration Analysis of the Forced Response of Rotating Cracked Blades," Proceedings of IMECE 2006, paper IMECE2006-15426, Chicago, Illinois, USA, November 2006.

${ }^{52}$ Powell, M. J. D., “A Hybrid Method for Nonlinear Equations," Numerical Methods for Nonlinear Algebraic Equations, edited by P. Rabinowitz, Gordon and Breach Science Publishers, London, 1970.

${ }^{53}$ Allemang, R. J., "The Modal Assurance Criterion - Twenty Years of Use and Abuse," Sound and Vibration, Vol. 37, 2003, pp. 14-23.

${ }^{54}$ Vakakis, A. F., Manevitch, L. I., Mikhlin, Y. V., Pilipchuk, V. N., and Zevin, A. A., Normal Modes and Localization in Nonlinear Systems, John Wiley \& Sons, Inc., New York, 1996. 